\title{
Vorarbeiten zur Entwicklung eines Immunoassays zur Schnelldiagnose von kardialem Troponin I bei Myokardinfarkten
}

\author{
Urs Hilbig ${ }^{1}$, Constanze Keck ${ }^{1}$ und Günter Gauglitz \\ ${ }^{1}$ Institut für Physikalische und Theoretische Chemie, Eberhard Karls Universität Tübingen, Tübingen, \\ Deutschland
}

Kontakt: urs.hilbig@uni-tuebingen.de

\section{Zusammenfassung:}

Erkrankungen des menschlichen Herz-Kreislaufsystem waren im Jahr 2011 die häufigste Todesursache in Deutschland [1]. Kardiales Troponin I ist ein hochrelevanter Marker bei Herzinfarkten. Die Reflektometrische Interferenzspektroskopie (RIfS) wurde als Messmethode gewählt. Es wurden verschiedene kommerziell erhältliche Antikörper auf ihre Eignung im Immunoassay hin getestet. Am Geeignetsten erwies sich der monoklonale Antikörper (B2268). Unterschiedliche Oberflächenimmobilisierungsstrategien wurden getestet und verglichen. Eine der Oberflächenimmobilisierungsstrategien in der Wasserkammer zeigt eine sehr geringe Standardabweichung und ausreichende Signalsteigung. Sie wird bei der weiteren Immunoassayentwicklung optimiert werden, um die bei einem Herzinfarkt relevanten Nachweißgrenzen zu erreichen.

\section{Schlagwörter:}

Biosensor, Troponin I, Immunoassay, POCT, Schnelldiagnose

\section{Motivation und Zielsetzung:}

Erkrankungen des menschlichen HerzKreislaufsystem waren im Jahr 2011 die häufigste Todesursache in Deutschland [1]. Ein nicht unerheblicher Anteil hiervon lässt sich auf Herzinfarkte zurückführen. Um eine individuell auf den Patienten abgestimmte Therapie durchzuführen, ist eine möglichst zeitnahe und valide Diagnose nach Auftreten eines Herzinfarktes von größter Bedeutung. Ergänzend zu den äußerlichen Symptomen des Herzinfarktes sollten hierzu klinische Parameter herangezogen werden. Bei kardialem Troponin I handelt es sich um einen hochrelevanten Marker, welcher sich für den Nachweis durch einen Immunoassay in einem Point-of-care-Testing (POCT) Device am Patientenbett sehr gut eignet. Die Vorarbeiten zur Immunoassayentwicklung für diesen Marker werden im Folgenden dargestellt.

\section{Methoden:}

Die Reflektometrische Interferenzspektroskopie:

Als Messmethode wurde die Reflektometrische Interferenzspektroskopie (RIfS) gewählt (Abb1.) [27]. Es handelt sich um eine direkt optische markierungsfreie Bestimmungsmethode. Nach erfolgter Kalibrierung können Assoziations- und Dissoziationsvorgänge zeitabhängig untersucht und dadurch Konzentrationen gemessen werden.

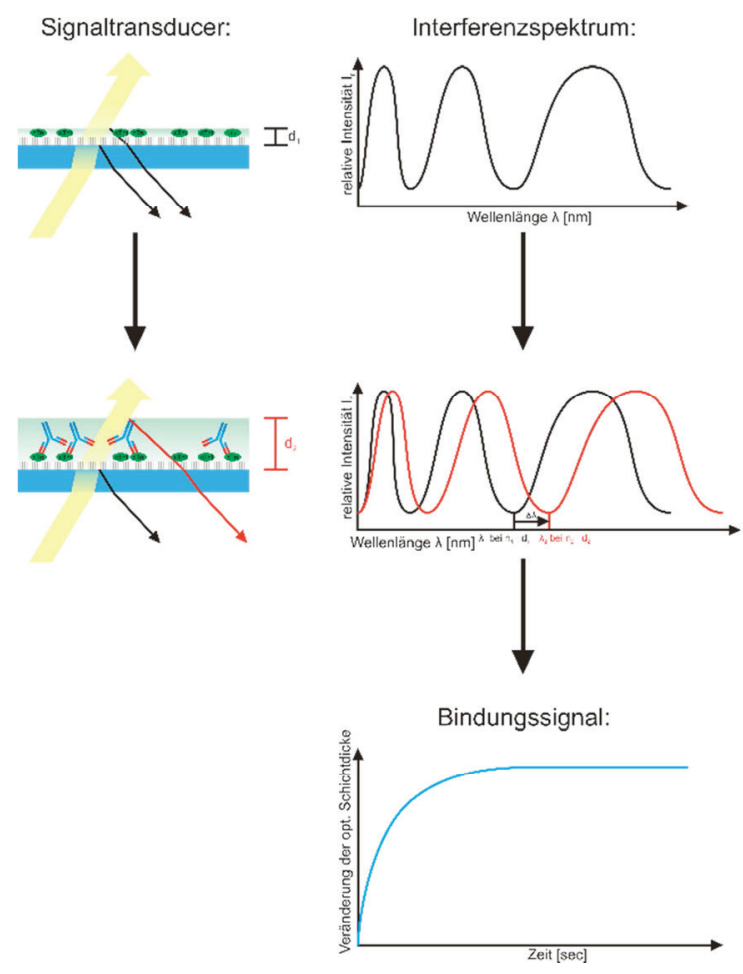

(A)

Abb. 1: RIfS Detektionsprinzp 


\section{Das RIfS Detektionsprinzip:}

Ein Glastransducer wird von Unten mit Weißlicht bestrahlt. Das entstandene Interferenzspektrum wird mittels eines Spektrometers ausgewertet (Abb.1: (A)). Lagert sich ein Analyt an den fixierten Ligand an, wird dies durch die Verschiebung eines Extremums des Interferenzspektrums detektierbar (Abb.1: (B)). Als Bindungssignal wird die relative Änderung der optischen Schichtdicke gegen die Zeit aufgetragen (Abb.1: (C)). Lässt sich der Analyt wieder vom Glastransducer entfernen, ist dies auch verfolgbar. In diesem Fall ist das System regenerierbar und kann für wiederholte Messungen eingesetzt werden. Im vorliegenden Immunoassay kann regeneriert werden. Es können also mehrere Messungen auf einem Transducer durchgeführt werden.

Ist die Transduceroberfläche gegenüber unspezifischen Wechselwirkungen abgeschirmt kann auch in für Immunoassays schwierigen Matrices wie Milch oder Seren gemessen werden [8-9]. Der hier entwickelte Assay soll später In Humanserum durchgeführt werden.

\section{Ergebnisse:}

Antikörperwahl:

Es wurden verschiedene kommerziell erhältliche polyklonale und monoklonale Antikörper auf ihre Eignung im Immunoassay hin getestet. Am Geeignetsten erwies sich der monoklonale Antikörper (B2268) in Kombination mit Troponin I (rekombinant, human).

Oberflächenvergleich:
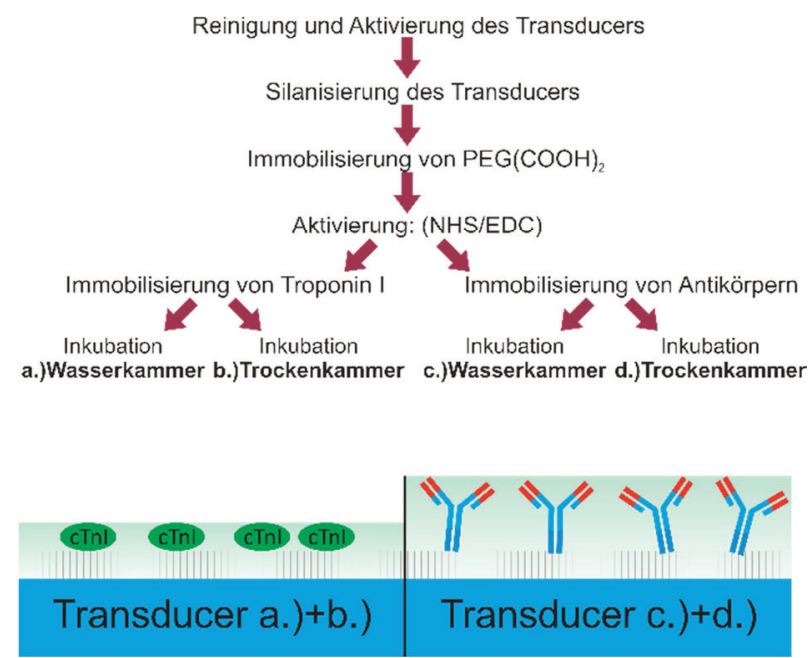

Abb. 2: Oberflächenimmobilisierungsstrategien
Wie in Abb. 2 gezeigt werden verschiedene Oberflächenimmobilisierungsstrategien getestet und verglichen. Es wurden sowohl das Antigen (Troponin I, cTnl) als auch der Antikörper immobilisiert, um verschiedene Assayformate testen zu können.

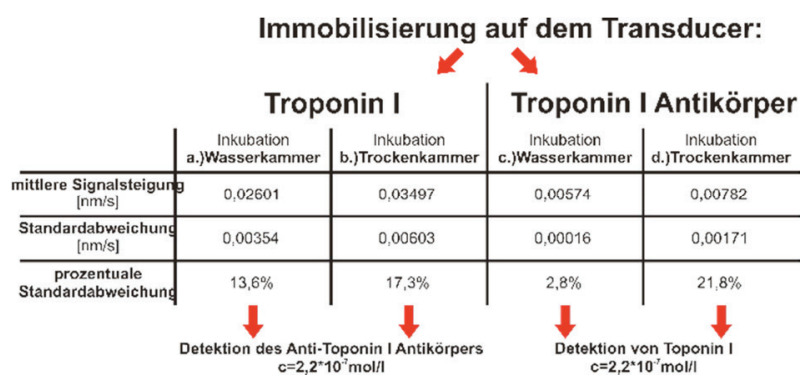

Abb. 3: Oberflächenvergleich

Abb.3 zeigt die Ergebnisse des Vergleichs der verschieden präparierten Transduceroberflächen.

Die Immobilisierungen in den trockenen Kammern (b und d) zeigen große Signale, aber auch hohe Standardabweichungen. Dies könnte zum einen auf eine Degradation der Proteine und die daraus resultierende höhere unspezifische Wechselwirkung zurückzuführen sein. Zum anderen auf einen Quelleffekt der trockenen Transduceroberfläche, welcher noch genauer untersucht werden wird.

Die Immobilisierungen in der Wasserkammer (a und c) zeigen kleinere Signale und geringere Standardabweichungen. Prinzipiell sind kleine Standardabweichungen und somit eine gute Reproduzierbarkeit wünschenswert. Mit dieser Transduceroberfläche wird der Assay weiterentwickelt werden.

Die Messungen wurden mit dem ausgewählten monoklonalen Antikörper (B2268) und Troponin I (rekombinant, human) durchgeführt. Alle Messungen wurden dreifach in PBS durchgeführt. Nach den Messungen wurde mit SDS 0,5\% pH=2 regeneriert.

\section{Diskussion und Ausblick:}

Erste Ergebnisse zeigen, dass ein monoklonaler Antikörper sich sehr gut für den Immunoassay eignet und eine ausreichende Wechselwirkung mit den präparierten Oberflächen zeigt. Es werden noch zusätzlich Versuche mit der Positivkontrolle (Troponin I Antigen) eines ELISAs gemacht werden, um die Eignung des Anti-Troponin I Antikörpers abschließend zu zeigen.

Eine der beiden Oberflächenimmobilisierungsstrategien in der Wasserkammer Abb.3 (c) zeigt 
eine sehr geringe Standardabweichung und ausreichende Signalsteigung. Mit dieser Oberfläche wird der Immunoassay weiterentwickelt.

Die Konzentrationen der gezeigten Messungen dienten dazu erste Eigenschaften des Systems kennenzulernen und sind über dem Detektionslimit, welches erreicht werden soll. Nach der Wahl des Antikörpers und der Oberflächenimmobilisierungsstrategie wird ein geeignetes Immunoassayformat gewählt werden. Weiter Oberflächenoptimierungen und unterschiedliche Regenerationsstrategien werden durchgeführt, um die erforderlichen Nachweisgrenzen mit dem Immunoassay zu erreichen.

\section{Literatur:}

[1] Statistisches Bundesamt: Todesursachen in Deutschland (2015)

[2] Brecht, A.; Gauglitz, G.; Nahm, W.: Interferometric measurements used in chemical and biochemical sensors. In: Analysis 20 (1992), Nr.3, S.135-140

[3] Schmitt, H.-M.; Brecht, A.; Piehler, J.; Gauglitz, G.: An integrated system for optical biomolecular interaction analysis. In: Biosens. Bioelectron. 12 (1997), Nr.80, S. 809-816

[4] Gauglitz, G.: Direct optical detection in bioanalysis: an update. In: Anal. Bioanal. Chem. 398 (2010), Nr.6, S. 2363-2372

[5] Piehler, J.; Brecht, A.; Gauglitz, G.; Zerlin, M.; Maul, C.; Thiericke, R.; Grabley, S.: Label-free monitoring of DNA-ligand interactions. In: Anal. Biochem. 249 (1997), Nr.1 S. 94-102

[6] Penttinen, P.; Jaehrling, J.; Damdmopoulos, A.E.; Inzunza, J.; Lemmen, J.G.; van der Saag, P.; Petterson, K.; Gauglitz, G.; Maekelae, S.; Pongratz, I.: Diet-derived polyphenol metabolite enterolactone is a tissue-specific estrogen receptor activator. In: Endocrinology 148 (2007), Nr.10, S. 4875-4886

[7] Rau, S.; Gauglitz, G.: Reflectometric interference spectroscopy (RlfS) as a new tool to measure in the complex matrix milk at low analyte concentration. In: Anal. Bioanal. Chem. 402 (2012), Nr.1, S. 529-536

[8] Rau, S.; Hilbig, U.; Gauglitz, G.: Label-free optical biosensor for detection and quantification of the nonsteroidal anti-inflammatory drug diclofenac in milk without any sample pretreatment. In. Anal. Bioanal. Chem. 406 (2014), Nr.14, S.3377-3386

[9] Ewald, M.; Le Blanc, A.F.; Gauglitz, G.; Proll, G.: A robust sensor platform for label-free detection of antiSalmonella antibodies using undiluted animal sera. In: Anal. Bioanal. Chem. 405 (2013), Nr.20, S.6461-6469 